\title{
Research on Robot Visual Servo Control Based on Image Identification
}

\author{
Qian $\mathrm{Wu}^{1,2, *}$ and Jinan $\mathrm{Gu}^{1}$ \\ ${ }^{1}$ Mechanical Information Research Center of Jiangsu Univ., Zhenjiang, 212013, China \\ ${ }^{2}$ Suzhou Vocational University, Suzhou, 215104, China \\ ${ }^{*}$ Corresponding author
}

\begin{abstract}
The research is mainly about robot system, DPS image processing system and Jacobian matrix. The robot in this study is a plenary robot with two rotary joints. It can only move in two directions, $x$ and $y$. The joints are powered by Panasonic ac servo motor. The CCD camera is installed at the end of the second arm. The math model of the robot visual servo system based on image was set up here. By avoiding the transformation of three-dimension coordinate, Jacobian matrix and its inverse matrix that show the relationship between the coordinate variation and the angle variation of the joints were set up. The two matrices were also used in the visual servo based on image. DSP image processing system processes the video image of object taken by CCD camera. It first transforms the image into gray level image. Then it gets the boundary of the round object after going through the processes of image binaryzation, median filtering and boundary extraction algorithm. Barycentric coordinates will be evaluated through point Hough transformation. The outcome will be sent to master control program of the Industrial Personal Computer through serial ports and thus the visual servo circulation is formed.
\end{abstract}

Keywords-robot; Jacobian matrix; DSP image processing system

\section{INTRODUCTION}

Great achievement has been scored in robot development since it came into being in the 1960s. Robot technology has played an important role and has been widely used in all walks of life such as industry, agriculture, service, national defense and spaceflight. It has freed man from complex and difficult work and improved the social productivity. All that have made the technology into an indispensable helper. With the development of industrial technology and the widened application of the robot, higher requirements such as higher adaptive capacity to environment and more intelligent decisive ability have been put forward on this technology.

\section{A. The Servo Control System of Image Identification}

The typical robot servo system consists of five parts: lighting, camera, image capture card, visual processor, and the robot itself. The keys of the normal operation of the robot visual servo system are the fast process ability and the design of the identification controller. The working process of the robot servo control system is as following: first transforming optical signal into electrical signal. Then transform it into digital image which can be directly processed by the visual processor through image capture card. The error between the present image in the above process and the expected image will be worked out by visual processor. The outcome will be mapped as the control signal, which will drive the joint robot to move. See Figure 1.

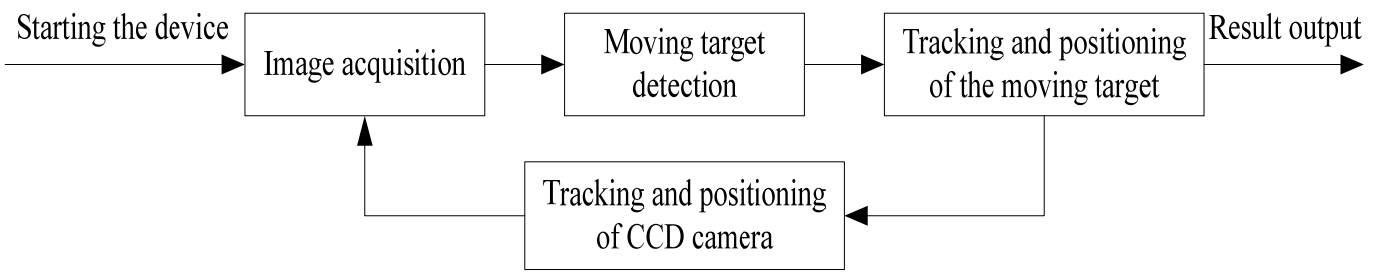

FIGURE I. ROBOT IDENTIFICATION CONTROL SYSTEM

\section{B. The Classification of the Robot Identification Control System}

(1)The Classification Based on Structure Classification. By the way of vision positioning installation, the camera is fixed at a certain position (like the right above or the lateral) in the robot space. In this way, fixed image resolution can be obtained, and at the same time, the image information of the mechanical arm and the work environment could also be achieved. This installation is convenient for the integration to the control system. The defects are: shade phenomenon of the image feature might happy in the process of robot motion, leading to less flexibility; the camera can not give detailed description of the task. Another kind is the eye-in-hand installation, which put the camera in the arm of the robot. Installation of this kind has a large vision and avoids the problem of image shade. Besides, the camera can come close the observed object with the adjustment of the arm position, 
and thus improves the image resolution and the measurement accuracy. The defect is the burden on the arm caused by the camera on it. Beside the camera is easy to break because of its location. What's more, it will be beyond the vision of the camera when coming close to the target.
(2)The Classification Based on Control Structure. Based on this, it can be divided into two kinds, double close-loop system and single close-loop system. The double close-loop identification system is shown as Figure 2.

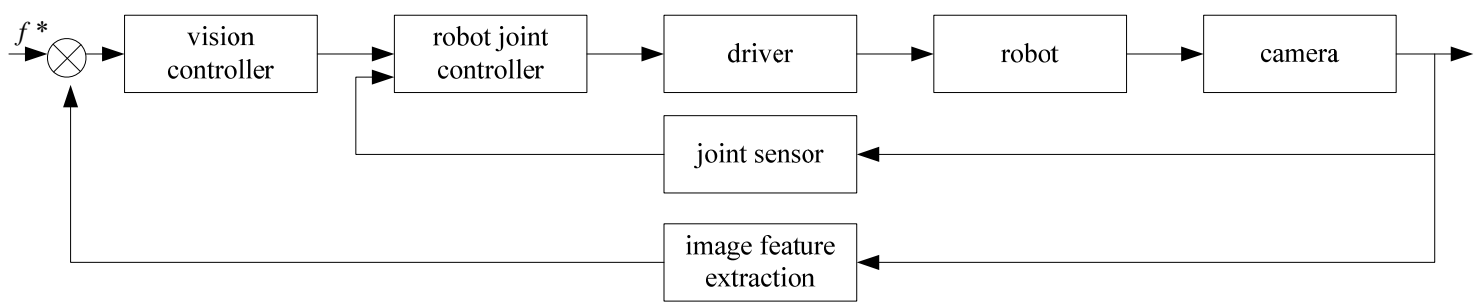

FIGURE II. DOUBLE CLOSE-LOOP IDENTIFICATION SYSTEM

Double close-loop system, also known as looks and move system, has two loops, the inner loop and the outer loop. The inner loop is called joint servo control, which can realize high speed sampling. The approximate linear robot feature can be obtained through by stabilizing the robot through the feedback of the its joint. The outer vision controller sets the angle of the joints with a low speed sampling. The double close-loop structure isolates mechanical movement singularity from its vision controller and takes robot as the ideal Cartesian motion original. It also simplifies the design process. Because most of the robots have reserve the port of receiving Cartesian datum speed or instruct of location increment, double close-loop system is easy to use and is widely adopted. The single close-loop identification control system is shown as Figure 3.

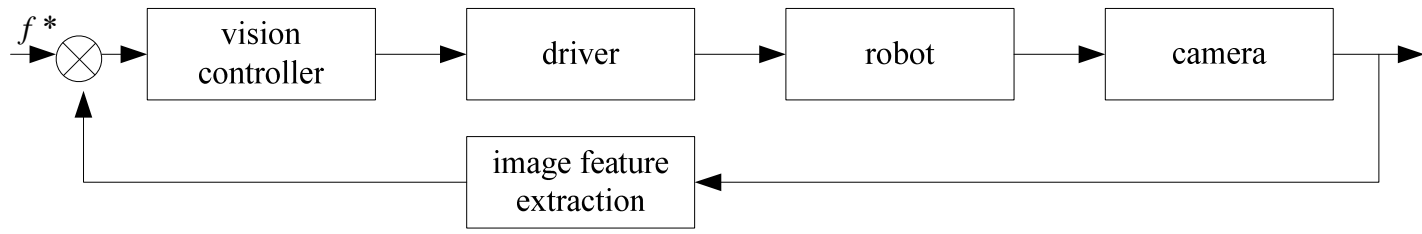

FIGURE III. SINGLE CLOSE-LOOP IDENTIFICATION CONTROL SYSTEM

\section{Robot Control Algorithm}

As for robot the problem of robot tracking and positioning of the moving target, widely researches have been carried out by foreign researchers. Proper algorithms have been put forward.

The key point of robot tracking and positioning lies in the following aspects: first, much relative information might lost while the image is projected from space to plane; second, the moving target in tracking process is a time-varying signal. Besides, in the process of tracking and positioning, target displacement, target rotation and image scaling also happen; third, in the process of tracking moving targets often shade each other. Similar appearance, frequent appearance and disappearance are also the factors having influence on the process. Given the above facts, researches on developing algorithm with high efficiency, high accuracy and stability remains to be the direction of machine vision development.

The background and significance of the research were introduced. It also talked about the components of the system, the classification of the robot vision control system, and the robot control algorithm.

\section{The BASIS OF Digital IMAGE}

Continues images are common. Discretization of the coordinate space and the property spaces of continues images should be conducted so as to process the images by computer.
Images discretized are called the digital images. Each unit of the digital images is called the picture element, simplified as pixel.

Certain relations exist between pixels. 4-neighborhood and 8 -neighborhood are the main components. The pixel $\mathrm{P}$, whose coordinate is $(\mathrm{x}, \mathrm{y})$, has 4 horizontal neighboring pixels and 4 vertical neighboring pixels. Their coordinates are $(\mathrm{x}+1, \mathrm{y}),(\mathrm{x}-1, \mathrm{y}),(\mathrm{x}, \mathrm{y}+1)$ and $(\mathrm{x}, \mathrm{y}-1)$ respectively. These pixel (represented by r) consist the 4-neighborhood, N (p), of p. See Table 1(a). The distance between pixel $\mathrm{p}$, whose coordinate is $(\mathrm{x}, \mathrm{y})$, and the its 4-neighborhood is 1 unit. The coordinates of the opposite angles' neighborhood pixel(represented by s) of pixel $\mathrm{p}$ are $(\mathrm{x}+1, \mathrm{y}+1),(\mathrm{x}+1, \mathrm{y}-1),(\mathrm{x}-1, \mathrm{y}+1)$ and $(\mathrm{x}-1, \mathrm{y}-1)$. They are written as $\mathrm{ND}(\mathrm{p})$. See Table 1 (b). The our pixel points together with the 4-neighborhood pixels of $p$ are called the 8-neighborhood of p, written as NS(p). See Table 1 (c).

TABLE I. THE NEIGHBORHOOD OF PIXEL

\begin{tabular}{|c|c|c|c|c|c|c|c|c|}
\hline & $\mathrm{r}$ & & $\mathrm{s}$ & & $\mathrm{s}$ & $\mathrm{s}$ & $\mathrm{r}$ & $\mathrm{s}$ \\
\hline $\mathrm{r}$ & $\mathrm{p}$ & $\mathrm{r}$ & & $\mathrm{p}$ & & $\mathrm{r}$ & $\mathrm{p}$ & $\mathrm{r}$ \\
\hline & $\mathrm{r}$ & & $\mathrm{s}$ & & $\mathrm{s}$ & $\mathrm{s}$ & $\mathrm{r}$ & $\mathrm{s}$ \\
\hline \multicolumn{3}{|c|}{$(\mathrm{a})$} & \multicolumn{3}{c|}{ (b) } & & (c) \\
\hline
\end{tabular}

\section{A. Image Binaryzation}

As for 8 bits gray image, each and every pixel is represented by an 8 -bit binary number. The image has 256 gray scales. The data value range is $0-255$. That is to say, the gray 
scales are represented by the integers ranging from 0 to 255 . The image binaryzation, or the thresholding, is an operation to indicate the images by two kinds of gray scales. As for the given threshold value $\mathrm{c}$, if the pixel value of this point is bigger than the threshold value, then value a will be taken as the new pixel value. Otherwise, the value $b$ will be taken as the pixel value. The formula is $\mathrm{y}=\mathrm{a}, \mathrm{y}>\mathrm{c}$ or $\mathrm{y}=\mathrm{b}, \mathrm{y} \leq \mathrm{c}$.

\section{B. Video Coding}

Digital image is the image that goes through digitalized conversion and can be represented and processed by digit. It is consist of pixel points. The number of the horizontal points is called horizontal resolution. The vertical one is called the vertical resolution. An integrated image has several lines, and is called a frame of image. The value of every pixel is different because of the different quantization precision. For example, selecting three 8-bit binary numbers with the same color component (the RGB method), and using the three 8-bit binary to represent one color point. $(200,100,64)$ described that the red component is 200, green is 100 and blue is 64 . The value of each component ranges from 0 to 256, representing the number of the scales is 256 (from the darkest to the lightest). Many static images can produce dynamic effect when continuously played. A speed of 25 frame/second or 30 frame/second can produce a smooth dynamic display.

\section{Color Space}

RGB color space: each and every pixel point is represented by $\operatorname{red}(R)$, Green $(G)$, and Blue $(B)$. The space is taken into usage in the task of image capture and display equipment, such as CCD, CMOS camera and CRT monitor. Because of the great correlation between different color components, the color space is not suitable for image processing. YCbCr color space: $\mathrm{Y}$ represents brightness, and $\mathrm{Cb}$ and $\mathrm{Cr}$ represent chromatic aberration. If the correlation is weak, do some proper operation. The color difference signal is sampled at 2:1 so as to save bandwidth.

\section{Video Image}

Movie is also moving image. By recording still images onto photographic films and displaying them one by one, we get the movie we see today. As for TV video, it has dozens of frames of still images. Each frame is composed of hundreds of lines. Each line has hundreds of pixel points. The number of frames of the image in 1 second is called frame count. The total number of lines in 1 second is called line frequency. The total number of the pixels is equal to video bandwidth.

\section{E. Jacobian Matrix}

The structure of Jacobian Matrix is based on visual servo based on image. It needs to be constructed in accordance with the feature of the image. Introduced by Weiss in 1987, Jacobian Matrix is used for describing the relations between the image feature space and the differential relationship of the position space of the robot arm end. The definition is as following:

$$
\dot{f}=J v(r) \dot{r}
$$

$$
J_{i}(r)=\left[\frac{\partial f}{\partial r}\right]=\left[\begin{array}{ccc}
\frac{\partial f_{1}(r)}{\partial r_{1}} & \cdots & \frac{\partial f_{1}(r)}{\partial r_{n}} \\
\vdots & \ddots & \vdots \\
\frac{\partial f_{m}(r)}{\partial r_{1}} & \cdots & \frac{\partial f_{m}(r)}{\partial r_{n}}
\end{array}\right]_{n \times n}
$$

Supposing the camera is at the end of the robot arm. In the above formula, $f \in R^{n \times n}$ represents the feature vector, $\dot{f}$ represents the rate of change of the relative image. $r \in R^{n}$ is the position vector of the robot end effectors in task space. $\dot{r}$ is the velocity vector of the robot end effectors. $J_{i}(r) \in R^{n \times n}$ is the Jacobian Matrix, which reflects the differentiable mapping relation between the robots moving space and the image feature space. The relation is also known as correlation matrix. In the differentiable mapping relations equation which tells the relation of actuator position change rate $\dot{r}$ and the angular velocity of robot joint $\dot{\theta}$. The equation is as following:

$$
\dot{r}=J_{r}(\theta) \cdot \dot{\theta}
$$

In the formula (3), $\dot{\theta} \in R^{p}$ is the angular velocity vector of the robot joint at $\mathrm{p}$ degree of freedom. $\dot{r} \in R^{n}$ is the velocity vector of the robot end effectors. $J_{r}(\theta) \in R^{n \times p}$ is the Jacobian Matrix.

$$
J_{r}(\theta)=\left[\frac{\partial r}{\partial \theta}\right]=\left[\begin{array}{ccc}
\frac{\partial r_{1}(\theta)}{\partial \theta_{1}} & \cdots & \frac{\partial r_{1}(\theta)}{\partial \theta_{p}} \\
\vdots & \ddots & \vdots \\
\frac{\partial r_{n}(\theta)}{\partial \theta_{1}} & \cdots & \frac{\partial r_{n}(\theta)}{\partial \theta_{p}}
\end{array}\right]_{n \times p}
$$

The relationship between robot feature space vector and robot joint angular speed vector can be obtained by formula:

$$
\begin{gathered}
\dot{f}=J_{i}(r) \cdot \dot{r}=J_{i}(r) \cdot J_{\theta}(\theta) \cdot \dot{\theta} \\
J=J_{i}(r) \cdot J_{\theta}(\theta)
\end{gathered}
$$

the formula 5 can be written into:

$$
\dot{f}=J \cdot \dot{\theta}
$$

$\mathrm{J}$ in the formula represents Jacobian matrix. It describes the proportion mapping relations between the joint vector and the speed change of the image feature. So the image feedback control can be designed in accordance with the differentiable mapping relationship between moving space of the robot joint and the image feature space.

DSP image process system and Jacobian matrix were studied. It includes the basis of digital image, binarization of 
image, the way of image decomposition, video coding and the component of Jacobian Matrix and its use.

\section{SUMMARY}

The thesis is mainly about how to set up the robot servo system based on image identification and how to realize servo service in the real robot system. The servo system has been set up after having a deep study on the robot servo theory and the components of the hardware. Proper mathematical models on robot visual servo were taken into use in the robot servo system. The robot servo system based on image identification has been initially realized.

The problem of robot servo system based on image identification is the key and difficult points in robot study. It is also a research area wealth studying. However, because the robot system is one has a strong feature of nonlinearity. It has strong joint coupling. With the increase of joints, the calculated quantity also has increased in the way of geometrical progression. Image processing is one discipline which needs great calculated quantity. High requirement has been raised on the hardware in order to guarantee the real-time performance and rapidity. The development of DSP image processing equipment has paved way for solving the problem. However, if combined with the visual servo robot, the study will be more difficult. Because of that, the development in this field remains in the stage of computer simulation. The practical servo control of the robot system has not been realized.

With the deepened study on the development of the robot servo system, more fruitful achievement will be scored for sure. I am convinced that intelligent robot will be realized in the near future.

\section{REFERENCES}

[1] Cheng Yunpeng.Matrix Theory[M].Xian : Northwestern Polytechnical University Press, 1999 .

[2] Sun Jixiang.Feature Extraction in Pattern Recognition and the Invariant Computer Vision [M].Beijing: National Defense IndustryPress, 2001.

[3] Xiang Longjiang.Model Independent Uncalibration Visual Servo Control [J].Robot, 2003, 25(5):424-427.

[4] Zang Yutong.A Research on Robot Servo Service Based on Image moment [D].Shenyang: Northeastern University, 2003.

[5] Tian Mengqian, Luo Xiang, Huang Weiyi.A Visual Servo Robot System Based on Uncalibrated Object Motion Estimation[J].Industrial Instrumentation and Automation,2004,2(1):29-32.

[6] Lin Jin.A Research on Robot Visual Servo Control System [D].Shanghai Tongji University, 1999.

[7] Zheng Nanning. Computer Vision and Pattern Recognition .National Defense Industry Press, 1998:20-85

[8] Yang Yanxi, Liu Ding, Yan Zhenjie.Simulation of Image Feedback Robot Manipulator Visual Servo System. Journal of System Simulation.2003,15(12):1737-1744

[9] Guo Xiaogin, Fei Yuenong, Xu Gang, Chen Jian.A Simulation System of Visual Servo Control of the Robotic Manipulator.Journal of Shenzhen University( Science and Engineering).2002,19(3) : 37-42

[10] Tian Mengqian, Luo Xiang, Huang Weiyi.A Research on Visual Servoing Robot To Manipulate Moving.Robot.2003,25(6):548-553

[11] Fu Jingxun, Yang Jingyu.Robotics.China Science and Technology Press, $1989: 1-40$

[12] Li Yushan.Digital Visual Video Technique .Xidian University Press,2005 : 35-150 